\title{
Experimental investigation of the influence of the passive porous coating on laminar-turbulent transition of the hypersonic boundary layer of the sharp cone at angles of attack
}

\author{
Sergey Morozov ${ }^{1, *}$, Sergey Lukashevich ${ }^{1}$ and Alexander Shiplyuk ${ }^{1}$ \\ ${ }^{1}$ Khristianovich Institute of Theoretical and Applied Mechanics SB RAS, 630090 Novosibirsk, Russia
}

\begin{abstract}
The problem of the delaying laminar to turbulent transition by the passive porous coating on the sharp cone at the angle of attack has been studied for the first time. Experiments are conducted in a Transit-M hypersonic short-duration wind tunnel at the Mach number 5.9. The boundary layer laminar-turbulent transition is determined by heat flux distribution on the surface of the cone. It is found that the transition on the porous surface in compare to smooth surface has been delaying on the windward side at the $0.5^{\circ}, 1^{\circ}$ and on leeward side at the $0.5^{\circ}$. There is no effect of the porous coating on the location of the laminar to turbulent transition at angle of attack $1^{\circ}$.
\end{abstract}

\section{Introduction}

Research of hypersonic boundary layers stabilization metods are an important problem of the hight speed aerodynamic due to the significant increase of the surface heat flux of the turbulent boundary layer in compare to the laminar boundary layer. The cause of the transition in hypersonic gradientless boundary layers is high frequencies unstable disturbances so called second mode disturbances [1]. On the passive porous coating with microstructure second mode disturbances grows slower than on the smooth surface [2]. The ability to delay transition by passive porous coatings are shown in several works [2 - 4].

The previous experimental studies are shown that it's possible either to reduce or magnify the energy of second mode disturbances using the porous coating with different length on the cone [11]. It indicates that the ability of the porous coating to delay transition is not predictable in case of small varying hypersonic boundary layer parameters. For instance, the stability of the second mode disturbances strongly depends on bluntness of the leading edge [6, 7, Ошибка! Источник ссылки не найден.] and angle of attack [9, 10].

Therefore, the present investigations are aimed at an experimental study of the efficiency of laminar to turbulent transition delay by the passive porous coating on a cone at angles of attack.

* Corresponding author: morozov@itam.nsc.ru 


\section{Experimental setup}

Experiments were carried out in the ITAM SB RAS hypersonic short-duration wind tunnel Tranzit-M at the freestream Mach number $\mathrm{M}_{\infty}=5.9$, stagnation temperature $\mathrm{T}_{0}=380 \pm 5 \mathrm{~K}$ and the unit Reynolds number $\operatorname{Re}_{1 \infty}=2 \cdot 10^{7} \mathrm{~m}^{-1}$.

The model is $7^{\circ}$ half-angle cone with sharp nose tip $\left(\mathrm{R}_{\text {bluntness }}=0.03 \mathrm{~mm}\right)$. The length of the cone generatrix is $363 \mathrm{~mm}$.

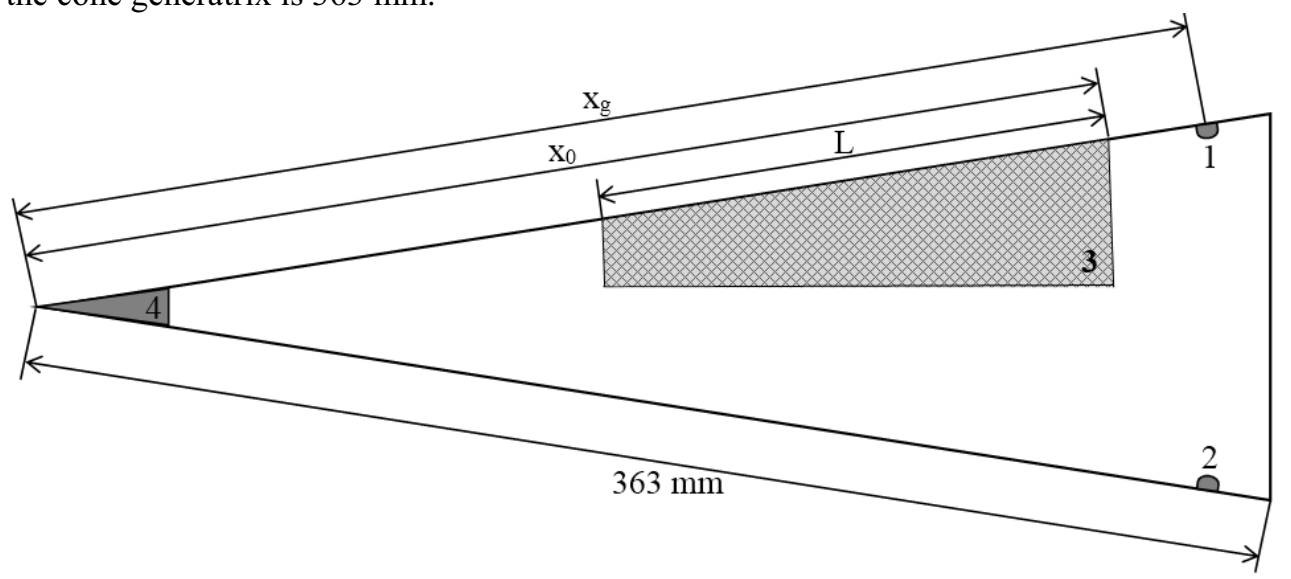

Fig. 1. Schematic of the model. 1, 2 - high frequency pressure gauges, 3 - passive porous coating, 4 interchangeable nose tip.

The coating is made of three layers of woven mesh made of nylon with a porosity of $44 \%$. The layers are turned with respect to each other by the angle of $45^{\circ}$. The coating thickness are chosen as most effective according to the experimental work [11]. The thickness equals to $0.18 \mathrm{~mm}$. The porous coating length is $210 \mathrm{~mm}$. The cone has been set at angles of attack $\alpha=0^{\circ}, 0.5^{\circ}, 1^{\circ}$.

The location of the boundary layer laminar-turbulent transition at the cone are determined by heat flux distribution along surface of the cone. Heat flux distributions are calculated from distributions of the surface temperature measured during the wind tunnel run by infrared camera FLIR SC7750L. Temperature measurement sampling rate is $200 \mathrm{~Hz}$.

\section{Results}

Fig. 2 shows the heat flux distribution along the smooth surface of the cone at zero angle of attack for $\operatorname{Re}_{1 \infty}=2 \cdot 10^{7} \mathrm{~m}^{-1}$. The boundary layer is laminar from $\mathrm{x}=57 \mathrm{~mm}$ to $\mathrm{x} \approx 100 \mathrm{~mm}$. The heat flux distribution deviations from linear law in log scales at $\mathrm{x} \approx 100 \mathrm{~mm}$ indicates the onset laminar to turbulent transition. The end point of the transition $x_{t r}=258 \mathrm{~mm}$ is determined at the location of the maximum of the heat flux distribution. Therefore, the boundary layer is transition at $\mathrm{x}=100 \div 258$ and turbulent after $\mathrm{x}_{\mathrm{tr}}=258$. 


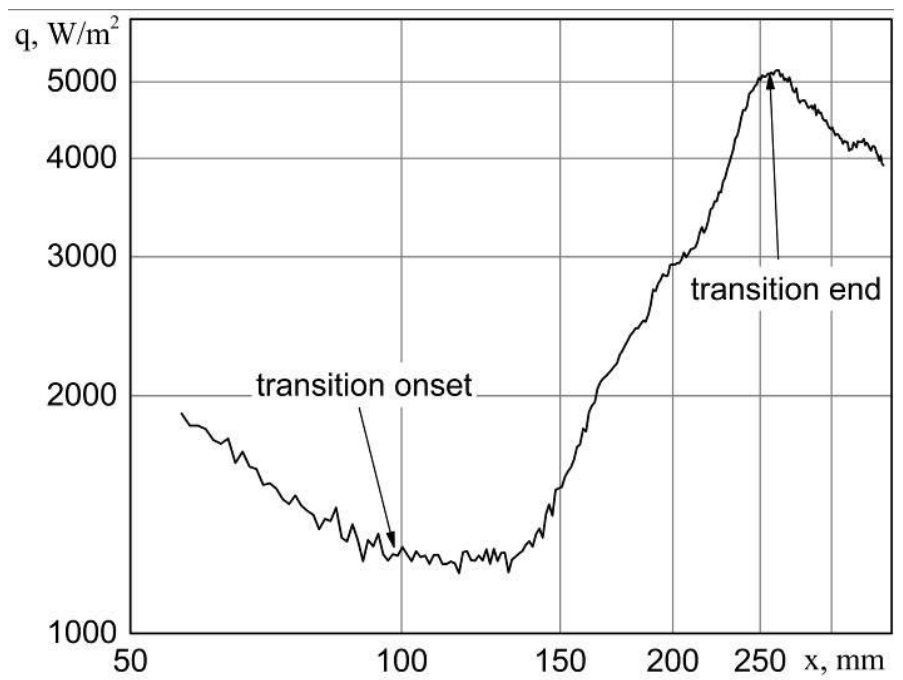

Fig. 2. The heat flux distribution on smooth surface of the cone at zero angle of attack for $\mathrm{Re}_{1 \infty}=$ $2 \cdot 10^{7} \mathrm{~m}^{-1}$.

The end point of the transition as function of the angle of attack for the unit Reynolds number $\operatorname{Re}_{1 \infty}=2 \cdot 10^{7} \mathrm{~m}^{-1}$ are shown in Figure 3. The laminar to turbulent transition on the smooth surface of the cone are shifted upstream on the leeward side and downstream at the windward side with increase of the angle of attack. The end point of the transition on the surface of the cone with passive porous coating are shifted further downstream at angles of attack $\alpha=-1 ;-0,5 ; 0 ; 0,5^{\circ}$ in compare with smooth surface. There is no transition delay at leeward side at $\alpha=1^{\circ}$. The onset of the transition in this case far downstream $(x=77 \mathrm{~mm})$ then the passive porous coating begins $(\mathrm{x}=120 \mathrm{~mm})$. Therefore, the porous coating has no stabilization effect on laminar and early transitional boundary layer.

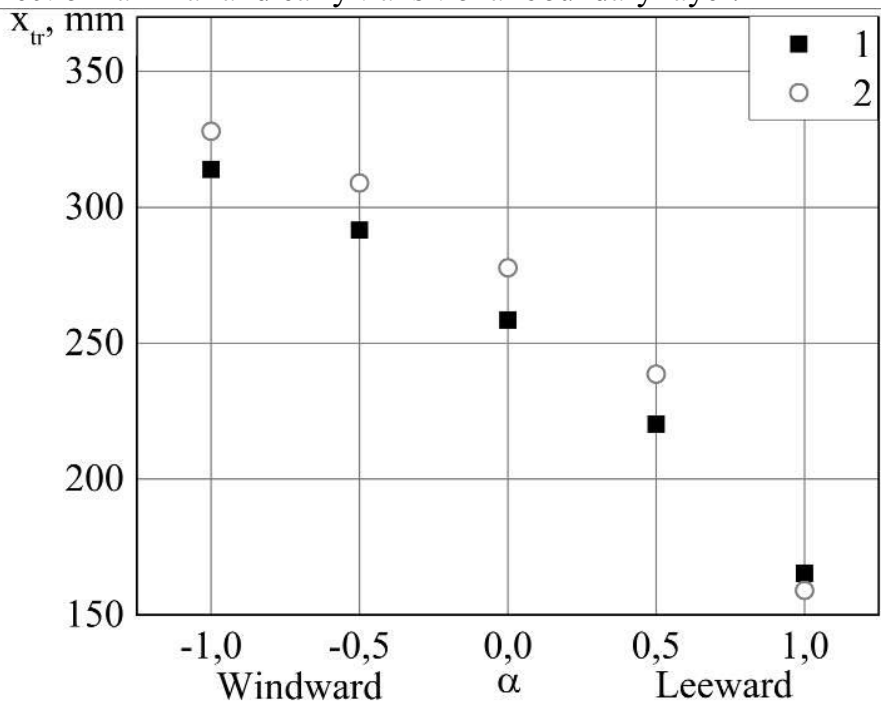

Fig. 3. The end point of the transition as function of the angle of attack for $\operatorname{Re}_{1 \infty}=2 \cdot 10^{7} \mathrm{~m}^{-1} .1-$ smooth surface; 2 - surface with passive porous coating. 


\section{Conclusions}

Experimental investigation of the influence of the passive porous coating on the laminarturbulent transition location on the sharp cone at angles of attack are performed at the freestream Mach number $\mathrm{M}_{\infty}=5.9$. The transition location is determined by measured heat flux distributions along smooth surface and surface with passive porous coating. The transition delay by passive porous coating is observed at angles of attack $\alpha=-1 ;-0,5 ; 0$; $0,5^{\circ}$, where minus sign means windward side. There is no transition delay at leeward side at $\alpha=1^{\circ}$ because the passive porous coating location in this case is no longer effective.

This work was financially supported by Russian Foundation for Basic Research (Grants No. 16-3800491 mol_a and No. 16-08-00965).

\section{References}

1. L.M. Mack, AIAA J. 13, 278 (1975)

2. A.V. Fedorov, N.D. Malmuth, A. Rasheed, H.G. Hornung, AIAA J. 39, 99 (2001)

3. A. Maslov, A. Shiplyuk, A. Sidorenko, P. Polivanov, A. Fedorov, V. Kozlov, N. Malmuth, AIAA Paper 1112, 1 (2006)

4. A. Wagner, M. Kuhn, J. M. Schramm, K. Hannemann, Exp. Fluids 54, 1606 (2013)

5. S.V. Lukashevich, S.O. Morozov, A.N. Shiplyuk, J. Appl. Mech. Techn. Phys. 54, 572 (2013)

6. K.F. Stetson, E.R. Thompson, J.C. Donaldson, L.G. Siler, AIAA Paper 0006, 1 (1984)

7. I. J. Lyttle, H. L. Reed, A. N. Shiplyuk, A. A. Maslov, D. A. Buntin, E. V. Burov, S. P. Schneider, AIAA J. 43, 1734 (2004).

8. A.A. Maslov, A.N. Shiplyuk, D.A. Bountin, A.A. Sidorenko, J. Spacecraft Rockets 43, 71 (2006)

9. K.F. Stetson, E.R. Thompson, J.C. Donaldson, L.G. Siler, AIAA Paper 0492, 1 (1985)

10. P. Balakumar, L.R. Owens, AIAA Paper 4718, 1 (2010)

11. S.V. Lukashevich, S.O. Morozov, A.N. Shiplyuk, Techn. Phys. Lett. 38, 1077 (2012) 